\title{
The best approach for sampling of pancreatic neuroendocrine tumors - EUS-FNA or EUS-FNB?
}

\section{다 (1) $\odot$}

\author{
Author \\ Per Hedenström \\ Institution \\ Division of Medical Gastroenterology, Department of \\ Internal Medicine, Sahlgrenska University Hospital, \\ Gothenburg, Sweden \\ Bibliography \\ DOI https://doi.org/10.1055/a-0959-6138 | \\ Endoscopy International Open 2019; 07: E1400-E1402 \\ (c) Georg Thieme Verlag KG Stuttgart · New York \\ eISSN 2196-9736
}

\author{
Corresponding author \\ Dr Per Hedenström, Medicinmottagningen, Sahlgrenska \\ Universitetssjukhuset, Blå Stråket 3, 41335 Göteborg, \\ Sweden \\ Fax: +4631827458 . \\ per.hedenstrom@vgregion.se
}

The limitations of transabdominal ultrasound in diagnosis of pancreatic diseases [1] were the driving force in developing endosonography (EUS) with the first echoendoscope being launched in 1980 [2]. The curvilinear array design of modern echoendoscope transducer heads enables EUS-guided sampling of lesions [3]. Traditionally, the principal sampling technique has been EUS-guided fine-needle aspiration (EUS-FNA) with open-tip needles designed for cytology [4].

Among all neoplasms originating from the pancreas, pancreatic neuroendocrine tumors (PanNETs) constitute a relatively rare entity. Incidence of PanNETs reportedly is increasing [5], and these tumors are challenging to diagnose with imaging alone [6], which implicates sampling of lesions suspected for PanNET. In addition, immunostaining for entity-specific tumor markers is required for reliable microscopic diagnosis [7].

Problematically, EUS-FNA is suboptimal in solid pancreatic lesions, with an $85 \%$ sensitivity for malignancy [8]. Furthermore, a majority of publications include mostly or exclusively pancreatic ductal adenocarcinomas $[9,10]$. The few studies addressing PanNETs have shown varying diagnostic sensitivity for EUS-FNA, ranging from $47 \%$ [11] to $90 \%$ [12], ( Fig. 1).

In recent years, a new generation of biopsy needles (EUSFNB [fine-needle biopsy]) has been developed for acquisition of whole tissue samples [13-15]. At present, it is not known whether FNB needles and processing of histology specimens can improve diagnosis of suspected PanNETs and motivate a shift from EUS-FNA.

In this issue of Endoscopy International Open, Eusebi et al contribute new knowledge on this important topic by investigating the diagnostic yield and sensitivity of EUS-FNB. The study has a retrospective design and it was conducted in a two-center setting during a 13-year period (2004-2017). Ex- clusively PanNETs were included and 102 EUS-guided sampling procedures were analyzed in 91 patients. Sampling was performed either by EUS-FNA (22/25-gauge needle), by EUS-FNB, or by both modalities. From 2004 to 2011, a 19-gauge QuickCore FNB-needle (Cook Medical, Limerick, Ireland) was used while using a 22/25-gauge reverse bevel ProCore FNB-needle (Cook Medical) or a 22-gauge opposing bevel SharkCore FNBneedle (Medtronic, Minneapolis, Minnesota, United States) from 2011 to 2017.

The authors report that the diagnostic yield, i. e. the acquisition of a macroscopically adequate sample, was $85 \%$ (35/41) in EUS-FNB and $78 \%$ (69/89) in EUS-FNA. In an intention-to-diagnose analysis, the final diagnostic sensitivity of EUS-FNB and EUS-FNA was $80 \%(33 / 41)$ and $69 \%(61 / 89)$, respectively. In dual sampling procedures $(n=28)$, the combination of EUSFNB and EUS-FNA had a significantly higher diagnostic yield than EUS-FNA alone, $96 \%(27 / 28)$ vs $75 \%$ (21/28), $P=0.023$. Either of the two techniques was diagnostic for PanNET in all of the 27 adequate samples. Seven EUS-FNA samples were inadequate for a conclusive diagnosis and in six of seven of these cases (86\%), the EUS-FNB sample was diagnostic. On the other hand, in six cases EUS-FNB was non-diagnostic and in all of these six cases, EUS-FNA was diagnostic. No noticeable difference in diagnostic performance was seen between the three FNB needles. No adverse events were recorded after EUS-FNB, which is a finding in line with the results of other studies [14, 16].

The study by Eusebi et al is important because a high number of patients were included and small PanNETs were not excluded. Moreover, few studies on EUS-FNB have been performed in cohorts containing exclusively PanNETs [17]. There are some weaknesses in the study discussed by the authors. As 


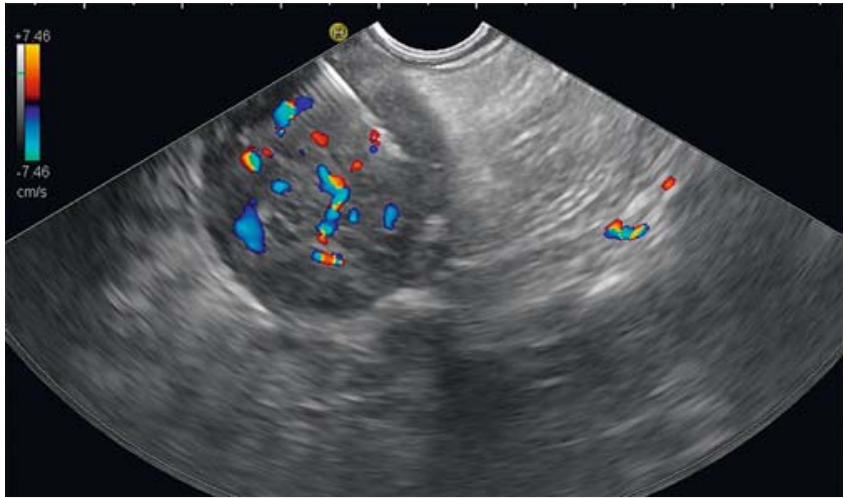

- Fig. 1 EUS-FNA of a hypoechoic, highly vascularized PanNET.

an example, different types of FNB needles were used, one of which - the Quick-Core needle - has been discarded by most endosonographers due to a high frequency of technical failures and a low diagnostic accuracy [18].

According to a recent study on solid pancreatic lesions [19], the accuracy of the reverse bevel FNB needle was found inferior (74\%) to that of the opposing bevel FNB needle (92\%). The number of cases sampled by EUS-FNB in the study by Eusebi and co-workers was not sufficient to determine which FNB needle is the superior one. Furthermore, there are yet other FNB needles available, such as the Franseen tip needle [20].

Importantly, the comparison of EUS-FNB and EUS-FNA is not exclusively a comparison between needles but rather a comparison between two different diagnostic approaches, which also include sampling maneuvers, sample preparation, and sample assessment by the (cyto)pathologist. Poor quality at any of these steps will result in a non-diagnostic work-up. This is a crucial aspect to keep in mind when interpreting studies investigating the accuracy of EUS-guided sampling.

Even though Eusebi and co-workers present valuable new data, it remains to be decided to what extent EUS-FNB may be superior to EUS-FNA in the work-up of suspected PanNETs. This study, like others [21], shows that EUS-FNB is a useful adjunct to EUS-FNA. Whether EUS-FNB should be used as the primary technique, or as a rescue technique after an unsuccessful EUSFNA, warrants further investigation. Studies analyzing the benefit of combining a 25-gauge FNA needle and a 22-gauge reverse bevel FNB needle in the same solid pancreatic lesion have shown contradictory results [21,22]. Moreover, such an approach implicates increased costs and a prolonged procedural time. Therefore, dual-modality sampling should be considered only in strictly selected cases. Future studies focusing on PanNETs should be designed as prospective, randomized trials using a predefined set of FNA and FNB needles with surgical specimens as the reference standard.

\section{Competing interests}

References

[1] DiMagno EP, Malagelada JR, Taylor WF et al. A prospective comparison of current diagnostic tests for pancreatic cancer. N Engl J Med 1977; 297: $737-742$

[2] DiMagno EP, Buxton JL, Regan PT et al. Ultrasonic endoscope. Lancet 1980; 1: 629-631

[3] Vilmann P, Khattar S, Hancke S. Endoscopic ultrasound examination of the upper gastrointestinal tract using a curved-array transducer. A preliminary report. Surg Endosc 1991; 5: 79-82

[4] Vilmann P, Hancke S. A new biopsy handle instrument for endoscopic ultrasound-guided fine-needle aspiration biopsy. Gastrointest Endosc 1996; 43: $238-242$

[5] Yao JC, Hassan M, Phan A et al. One hundred years after "carcinoid": epidemiology of and prognostic factors for neuroendocrine tumors in 35,825 cases in the United States. J Clin Oncol 2008; 26: 3063-3072

[6] Delbeke D, Pinson CW. Pancreatic tumors: role of imaging in the diagnosis, staging, and treatment. J Hepatobiliary Pancreat Surg 2004; 11: $4-10$

[7] Falconi M, Bartsch DK, Eriksson B et al. ENETS Consensus Guidelines for the management of patients with digestive neuroendocrine neoplasms of the digestive system: well-differentiated pancreatic nonfunctioning tumors. Neuroendocrinology 2012; 95: 120-134

[8] Hewitt MJ, McPhail MJ, Possamai L et al. EUS-guided FNA for diagnosis of solid pancreatic neoplasms: a meta-analysis. Gastrointest Endosc 2012; 75: 319-331

[9] Alatawi A, Beuvon F, Grabar S et al. Comparison of 22G reverse-beveled versus standard needle for endoscopic ultrasound-guided sampling of solid pancreatic lesions. United Eur Gastroenterol J 2015; 3: $343-352$

[10] Hebert-Magee S, Bae S, Varadarajulu S et al. The presence of a cytopathologist increases the diagnostic accuracy of endoscopic ultrasound-guided fine needle aspiration cytology for pancreatic adenocarcinoma: a meta-analysis. Cytopathology 2013; 24: 159-171

[11] Voss M, Hammel P, Molas G et al. Value of endoscopic ultrasound guided fine needle aspiration biopsy in the diagnosis of solid pancreatic masses. Gut 2000; 46: 244-249

[12] Atiq M, Bhutani MS, Bektas M et al. EUS-FNA for pancreatic neuroendocrine tumors: a tertiary cancer center experience. Dig Dis Sci 2012; 57: $791-800$

[13] Bang JY, Hebert-Magee S, Navaneethan U et al. EUS-guided fine needle biopsy of pancreatic masses can yield true histology: results of a randomised trial. Gut 2018; 67: 2081 -2084

[14] Iglesias-Garcia J, Poley JW, Larghi A et al. Feasibility and yield of a new EUS histology needle: results from a multicenter, pooled, cohort study. Gastrointest Endosc 2011; 73: 1189-1196

[15] Jovani M, Abidi WM, Lee LS. Novel fork-tip needles versus standard needles for EUS-guided tissue acquisition from solid masses of the upper GI tract: a matched cohort study. Scand J Gastroenterol 2017; 52: $784-787$

[16] Bang JY, Hebert-Magee S, Trevino J et al. Randomized trial comparing the 22-gauge aspiration and 22-gauge biopsy needles for EUS-guided sampling of solid pancreatic mass lesions. Gastrointest Endosc 2012; 76: $321-327$

[17] Witt BL, Factor RE, Chadwick BE et al. Evaluation of the SharkCore((R)) needle for EUS-guided core biopsy of pancreatic neuroendocrine tumors. Endoscopic ultrasound 2018; 7: 323-328

[18] Fernandez-Esparrach G, Sendino O, Sole M et al. Endoscopic ultrasound-guided fine-needle aspiration and trucut biopsy in the diagnosis of gastric stromal tumors: a randomized crossover study. Endoscopy 2010; 42: $292-299$ 
[19] Nayar MK, Paranandi B, Dawwas MF et al. Comparison of the diagnostic performance of 2 core biopsy needles for EUS-guided tissue acquisition from solid pancreatic lesions. Gastrointest Endosc 2017; 85: $1017-1024$

[20] Mitri RD, Rimbas M, Attili F et al. Performance of a new needle for endoscopic ultrasound-guided fine-needle biopsy in patients with pancreatic solid lesions: A retrospective multicenter study. Endosc Ultrasound 2018; 7: 329-334
[21] Hedenstrom P, Demir A, Khodakaram K et al. EUS-guided reverse bevel fine-needle biopsy sampling and open tip fine-needle aspiration in solid pancreatic lesions - a prospective, comparative study. Scand J Gastroenterol 2018; 53: 231-237

[22] Berzosa M, Villa N, El-Serag HB et al. Comparison of endoscopic ultrasound guided 22-gauge core needle with standard 25-gauge fineneedle aspiration for diagnosing solid pancreatic lesions. Endosc Ultrasound 2015; 4: 28-33 